\title{
MINIMAL FUNCTIONS, MARTINGALES, AND BROWNIAN MOTION ON A NONCOMPACT SYMMETRIC SPACE
}

\author{
J. C. TAYLOR
}

\begin{abstract}
A Brownian motion on $\mathbf{R}^{n}$ may be characterized as a process $\left(X_{t}\right)_{t \geqslant 0}$ on a probability space $(\Omega, \mathfrak{F}, P)$ such that, for all $y \in \mathbf{R}^{d}, \exp \left\{-(t / 2)\|y\|^{2}+\left\langle y, X_{t}\right\rangle\right\}$ is a martingale of expectation one. The analogue of this fact is proved for the Brownian motion on a noncompact symmetric space.
\end{abstract}

Introduction. A Brownian motion on $\mathbf{R}^{n}$ started from zero may be characterized as a process $\left(X_{t}\right)_{t \geqslant 0}$ on a probability space $(\Omega, \mathfrak{F}, P)$, adapted to a filtration $\left(\mathfrak{F}_{t}\right)_{t \geqslant 0}$, for which the processes $M(u)=\left(M_{t}(u)\right)_{t \geqslant 0}$, where $M_{t}(u)=\exp \left\{-(t / 2)\|y\|^{2}+\right.$ $\left.\left\langle y, X_{t}\right\rangle\right\}$, are all martingales of expectation one. These processes are obtained by composing the space-time process with the minimal solutions

$$
u(x, t)=\exp \left\{-(t / 2)\|y\|^{2}+\langle y, x\rangle\right\}
$$

of the heat equation $\Delta u+2 u_{t}=0$ on $\mathbf{R}^{n} \times \mathbf{R}$.

The purpose of this note is to show that the same result holds for the Brownian motion on any noncompact symmetric space. One natural question to ask is whether this is a very general phenomenon. It will be shown in a subsequent article that for the invariant diffusion on the Heisenberg group the theorem is false. In this case knowing that martingales are produced only implies that the component of this diffusion in the corresponding $\mathbf{C}^{n}$ is a standard Brownian motion. It is not known whether the theorem is valid for uniformly elliptic operators on $\mathbf{R}^{n}$.

The author thanks the referee for clarifying the exposition by pointing out the key role of Proposition 3.4.

1. The main result. Let $(\Omega, \mathfrak{F}, P)$ be a probability space with an increasing filtration $\left(\mathfrak{F}_{t}\right)_{t \geqslant 0}$ and let $\left(X_{t}\right)_{t \geqslant 0}$ be an adapted stochastic process on $(\Omega, \mathfrak{F}, P)$ valued in a noncompact symmetric space $X=G / K$, where $G$ is a semisimple Lie group and $K$ is a maximal compact subgroup.

THEOREM. The following statements are equivalent:

(1) the finite dimensional joint distributions of the process $\left(X_{t}\right)_{t \geqslant 0}$ are the same as those of Brownian motion on $X$ started from zero (the coset $K$ ); in other words, the process is equivalent to this Brownian motion;

Received by the editors May 1, 1986.

1980 Mathematics Subject Classification (1985 Revision). Primary 60H99; Secondary 43A85.

The author was supported by NSERC Operating Grant \# A3108.

(C1987 American Mathematical Society $0002-9939 / 87 \$ 1.00+\$ .25$ per page 
(2) for every minimal solution $u$ of the heat equation $\Delta u+2 u_{t}=0$ on $X \times \mathbf{R}$, the process $M(u)=\left(M_{t}(u)\right)_{t \geqslant 0}$ is an $\left(\mathfrak{F}_{t}\right)$-martingale with expectation 1 , where $M_{t}(u)=$ $u\left(X_{t}, t\right)$ for all $t \geqslant 0$.

Proof. (1) $\Rightarrow(2)$ Let $\left(P_{t}\right)_{t \geqslant 0}$ be the transition semigroup for Brownian motion on $X$. It follows from the uniqueness of the positive Cauchy problem on $X$ [7], that for every $u \geqslant 0$, a solution of the heat equation $\Delta u=-2 u_{t}$ on $X \times(a, b)$, $\int P_{t}(x, d y) u(y, s)=u(x, s-t)$, if $a<s-t<s<b$. As a result, $M(u)$ is a martingale if $u$ is a nonnegative solution on $X \times \mathbf{R}$. From the formulas given below for the minimal solutions, it is clear that $M_{0}(u)=1$.

Before proving the converse, i.e. $(2) \Rightarrow(1)$, it is useful to set up the necessary analytic machinery on $X$ for characterizing probabilities. Those not familiar with symmetric space terminology should take $X$ as the hyperbolic disc for which the details are presented in a straightforward fashion in Helgason [5]. For details on symmetric spaces see Helgason [4].

2. Determining a probability on $X$. Let $X=G / K$, where $G$ is a semisimple Lie group and $K$ is a maximal compact subgroup. Let $G=K A N$ be an Iwasawa decomposition of $G$. Since $a N a^{-1} \subseteq N$ for all $a \in A$, it follows that the set $A N=N A=S$ is a subgroup, in fact a semidirect product of the abelian group $A$ with the nilpotent group $N$. The group $A$ is $\cong \mathbf{R}^{r}$, where $r$ is the rank of $X$.

Let $o$ denote the coset $K$. The horocycles of $X$ are the orbits of the groups $g \mathrm{Ng}^{-1}$ in $X$. They may be arranged in families of parallel horocycles: all the horocycles $k a(N \cdot o)=k a N(k a)^{-1} \cdot(k a \cdot o), a \in A$, belong to the same parallel family of horocycles normal to the direction $k M=b$ in the Furstenberg boundary $B=K / M$ (cf. Helgason [2]). If $g \cdot o=x \in X$, then for each $k M=b \in B$, there is a unique horocycle $\xi(x, b)$ that contains $x$ and is normal to $b$. It is the horocycle $\xi=$ $k a(N \cdot o)$, where $a=a(x, b)=\exp A(x, b)$, i.e. $A(x, b)$ is the logarithm of the $A$-component of the NAK-Iwasawa decomposition of $k^{-1} g$ if $x=g \cdot o$. (In the case of the hyperbolic disc, $A(x, b)$ is denoted in [5] by $\langle z, b\rangle$.) When considering the horocycles $\xi(x, b)$ one is viewing $X$ as isomorphic to the group $k S k^{-1}$ under the map kank $^{-1}=g \rightarrow g \cdot o=k a n \cdot o$.

In [3], Helgason defined the Radon-Fourier transform $\tilde{f}$ of a function $f \in C_{c}^{\infty}(X)$ by the formula

$$
\tilde{f}(\lambda, b)=\int_{X} f(x) \exp \{(-i \lambda+\rho)(A(x, b))\} d x
$$

where $\lambda \in a^{*}$ and $b \in B=K / M$. He proved in Theorem 2.2 of [3] that

$$
f(X)=\int_{a^{*} \times B} \tilde{f}(\lambda, b) \exp \{(i \lambda+\rho)(A(x, b))\}|c(\lambda)|^{-2} d \lambda d b .
$$

As a result, it follows that each $f \in C_{c}^{\infty}(X)$ may be continuously decomposed into plane waves $f_{b}(x)$, that is $f(x)=\int_{B} f_{b}(x) d b$, where

$$
f_{b}(x)=\int_{\mathfrak{a}^{*}} \tilde{f}(\lambda, b) \exp \{(i \lambda+\rho)(A(x, b))\}|c(\lambda)|^{-2} d \lambda
$$

is constant on the horocycles normal to $b$. 
Since $A(o, b)=0$ implies that $C_{b}=\int_{a^{*}}|\tilde{f}(\lambda, b)||c(\lambda)|^{-2} d \lambda<\infty$, it follows that $\left|f_{b}(x)\right| \leqslant C_{b} \exp \rho(A(x, b))$, where $C_{b}$ varies continuously.

As an immediate corollary of these formulas one has

LEMMA 2.1. Let $\mu$ be a probability on $X$. For each $b \in B$ let $\mu_{b}$ be the measure on $A$ $\left(\simeq \mathbf{R}^{r}\right.$ ) defined as follows: if $C$ is a Borel set in $A$, let $\mu_{b}(C)=\mu$ (the union of all horocycles normal to $b$ and meeting $C \cdot o=C K)$. Assume that

$$
\text { for any constant } K \in \mathbf{R} \text { and } b \in B, K \exp \rho(A(x, b)) \in L^{1}(\mu) \text {. }
$$

Then

$$
\begin{aligned}
\int f(x) \mu(d x) & =\int_{B} \int f_{b}(x) \mu(d x) d b \\
& =\int_{B} \int_{A} f_{b}(a \cdot o) \mu_{b}(d a), \text { where } a=A(x, b) .
\end{aligned}
$$

Consequently, if $\mu$ satisfies (*) the family of measures $\mu_{b}, b \in B$, determines $\mu$.

REMARK. Since any root space $\mathfrak{g}_{\alpha}$ is orthogonal under the Killing form to $\mathfrak{g}_{o}$, the horocycle $N \cdot o$ is orthogonal to $A \cdot o$ at $o$. As a result, if $d(x, y)$ is the geodesic distance of $x$ to $y$, then $d(o, a n \cdot o) \geqslant d(o, a \cdot o)$ for any $n \in N$. Hence, condition $(*)$ may be replaced by the stronger condition

$$
\text { for all } C>0, \exp C d(o, x) \in L^{1}(\mu) \text {. }
$$

3. The rest of the proof. Firstly, one shows that if the minimal solutions $u$ of the heat equation $\Delta u+2 u_{t}=0$ on $X \times \mathbf{R}$ give rise to martingales, then so does any nonnegative solution. This merely amounts to giving a measurable parametrization of the set of minimal solutions.

In view of the results of Karpelevic [6] and Koranyi and Taylor [7], the set of minimal solutions of the heat equation on $X \times \mathbf{R}$ is the set of functions

$$
u_{m}(x, t)=e^{-c t / 2}+(\lambda+\rho)(A(x, b)),
$$

where $m=(b, \lambda, c) \in K / M \times \Lambda_{c} \times\left[-\|p\|^{2},+\infty\right)$ is determined by $b=k M ; \lambda$, $\rho \in a^{*}$, and $\lambda$ corresponds, under the duality determined by the Killing form, to a point in the closure of $\mathfrak{a}^{+}$of length $\left(\|\rho\|^{2}+c\right)^{1 / 2}$ (i.e. $\Lambda_{c}$ is identified with the intersection of the closure of $a^{+}$with the sphere of radius $\left.\left(\|\rho\|^{2}+c\right)^{1 / 2}\right)$; and $c=\|\lambda\|^{2}-\|\rho\|^{2}$. Let $D$ denote the parameter set $K / M \times \Lambda_{c} \times\left[-\|\rho\|^{2},+\infty\right)$ and let $\mathfrak{D}$ be the Borel $\sigma$-field on $D$. Clearly, if $\mathfrak{X}$ is the Borel $\sigma$-field on $X$, for each $t$, $(m, x, t) \rightarrow u_{m}(x, t)$ is $\mathfrak{D} \times \mathfrak{X}$-measurable as it is even $C^{\infty}$.

To each solution $u \geqslant 0$ of the heat equation $\Delta u=2 u_{t}$ on $X \times \mathbf{R}$, there corresponds a unique measure $\mu$ on $D$ such that $u(x, t)=\int u_{m}(x, t) \mu(d m)$. The hypothesis (2), that the minimal solutions $u$ give rise to martingales, and Fubini's theorem imply the validity of

LEMMA 3.1. For any nonnegative solution $u$, the process $M(u)$ is a martingale.

The Laplace-Beltrami operator has a very simple form when acting on functions $f(x), x=g \cdot o$, that depend only on the $A$-component in the NAK-Iwasawa decomposition of $k^{-1} g$. 
LEMMA 3.2 (CF. [6], ALSO [5, p. 267]). Let $b=k M \in B$ and let $f$ be a function that depends only on $a(x, b)$, i.e. $f(k a n \cdot o)=f(k a \cdot o)$ for all $a \in A$ and $n \in N$. If $H=A(x, b)$, let $\psi(H)=f(k \exp H \cdot o)$. Then

$$
\Delta f(x)=e^{\rho(H)} \Delta_{\mathrm{a}}\left(\psi e^{-\rho}\right)(H)-\|\rho\|^{2} \psi(H),
$$

where $\Delta_{\mathfrak{a}}$ is the Laplacian on a determined by the inner product given by the Killing form.

COROllary 3.3. For every $\lambda \in a^{*}$ of length $\left(\|\rho\|^{2}+c\right)^{1 / 2}$, the function $u=$ $\exp \{-c t / 2+(\lambda+\rho)(A(x, b))\}$ is a nonnegative solution of the heat equation on $X \times \mathbf{R}$.

REMARK. This collection of functions contains all the minimal solutions and many others.

As a result of Lemma 3.1 one has the following key result.

Proposition 3.4. There is a unique probability $\mu_{t}$ on $X$ such that for all $\lambda \in \mathfrak{a}$ and $b \in B$,

$$
\int \exp (\lambda+\rho)(A(x, b)) \mu_{t}(d x)=\exp (\lambda+\rho)\left(A\left(x_{0}, b\right)\right) \exp \left\{\left(\|\lambda\|^{2}-\|\rho\|^{2}\right)\right\} t / 2
$$

Further, this probability is the law of Brownian motion at time $t$ when started from $x_{0}$. When $x_{0}=0$, this simplifies to

$$
\int \exp (\lambda+\rho)(A(x, b)) \mu_{t}(d x)=\exp \left\{\left(\|\lambda\|^{2}-\|\rho\|^{2}\right)\right\} t / 2
$$

as $A(o, b)=0$ for all $b \in B$.

Proof. It has been shown that if $\left(y_{t}\right)_{t \geqslant 0}$ is a Brownian motion started from any point, then any nonnegative solution of the heat equation gives rise to a martingale. As a result, if $\mu_{t}$ is the law of $y_{t}$, then by Corollary 3.3, it satisfies (*).

It follows from Lemma 2.1 that any probability $\nu$ on $X$ that satisfies $(*)$ is determined by the probabilities $\nu_{b}, b \in B$. Since

$$
\int \exp (\lambda+\rho)(A(x, b)) \nu(d x)=\int \exp (\lambda+\rho)(A(x, b)) \nu_{b}(d x),
$$

(*) gives the Laplace transforms of these probabilities, viewed as probabilities on $\mathbf{R}^{r}$, it is completely determined, and so $\nu=\mu_{t}$.

REMARK 3.5. If one fixes $b \in B$ and considers the martingales given by the list of functions in Corollary 3.3 it is easy to see that the process $\left(H_{t}\right)_{t \geqslant 0}=\left(A\left(X_{t}, b\right)\right)_{t \geqslant 0}$ is a stationary Gaussian process on $a \cong \mathbf{R}^{r}$, with independent increments and initial position 0 . The law of $H_{t}-H_{s}$ is

$$
(2 \pi(t-s))^{-r / 2} \exp \left\{-\left\|H+(t-s) H_{\rho}\right\| / 2(t-s)\right\} d H,
$$

where $H_{\rho} \in$ a corresponds to $\rho$ under the duality given by the Killing form. This result for the Brownian motion is due to Karpelevic [6]. 
Once one knows that the 1-dimensional distributions of the process $\left(X_{t}\right)_{t \geqslant 0}$ are those of Brownian motion started from $o$, the remaining finite-dimensional distributions can be computed using Proposition 3.4 and regular conditional probabilities. Assume for simplicity's sake that the process itself has regular conditional probabilities $\pi_{t, s}(\omega, \Gamma)$, i.e.

$$
E\left[f\left(X_{t}\right) \mid \mho_{s}\right]=\int \pi_{s, t}(\cdot, d \sigma) f(\sigma) \quad P \text {-a.s. }
$$

Let $f(x)=\exp (\lambda+\rho)(A(x, b))$. It follows from Corollary 3.3 that

$$
\begin{aligned}
& \int \pi_{s, t}(\omega, d \sigma) \exp (\lambda+\rho)(A(\sigma, b)) \\
& \quad=\exp (\lambda+\rho)\left(A\left(X_{s}(\omega), b\right)\right) \exp \left\{\left(\|\lambda\|^{2}-\|\rho\|^{2}\right)(t-s) / 2\right\} \quad P \text {-a.s. }
\end{aligned}
$$

From Proposition 3.4 it follows that $P$-a.s. $\pi_{t, s}(\omega, d \sigma)$ is the law of Brownian motion at time $(t-s)$ started from $X_{s}(\omega)$. Consequently, $E\left[f\left(X_{t}\right) \mid \mathfrak{F}_{s}\right]=$ $E\left[f\left(X_{t}\right) \mid X_{s}\right]$ and so the process is Markov, cf. [1]. As a result one may compute the finite-dimensional joint distributions by an obvious induction: if $0<t(1)<t(2)<$ $\cdots<t(n)=s<t(n+1)=t$ and $f, f_{i}, 0 \leqslant i \leqslant n$, are in $\complement_{o}^{\infty}(X)$, then

$$
E\left[\prod_{i} f_{i}\left(X_{t(i)}\right) f\left(X_{t}\right)\right]=E\left[\prod_{i} f_{i}\left(X_{t(i)}\right) E\left[f\left(X_{t}\right) \mid X_{s}\right]\right] \text {. }
$$

REMARK 3.6. It is clear that any process satisfying (1) of the theorem is equivalent to one with continuous paths and so to one with regular conditional probabilities. However, if one only assumes (2) it is not a priori obvious that the process is equivalent to one with regular conditional probabilities. Without assuming this, one may still prove that $(2) \Rightarrow(1)$ by reasoning with the finite-dimensional joint distributions. Let $0<t(1)<t(2)<\cdots<t(n)=s<t(n+1)=t$ and let $\nu$ be the joint distribution of $\left(X_{0}, X_{t(1)}, X_{t(2)}, \ldots, X_{s}, X_{t}\right)$. If $\left(x, x_{n+1}\right) \in X^{n+1} \times X$ let $\pi\left(x, d x_{n+1}\right)$ be a regular conditional probability for $\nu$ given $x$. Let

$$
f\left(x_{0}, x_{1}, x_{2}, \ldots, x_{n}, x_{n+1}\right)=\exp \left\{-c t / 2+(\lambda+\rho)\left(A\left(x_{n+1}, b\right)\right)\right\} .
$$

The martingale condition implies that for $x=x(\omega)=\left(X_{0}, X_{t(1)}, X_{t(2)}, \ldots, X_{s}\right)$,

$$
\begin{aligned}
& \int \pi\left(x(\omega), d x_{n+1}\right) \exp (\lambda+\rho)\left(A\left(x_{n+1}, b\right)\right) \\
& \quad=\exp (\lambda+\rho)\left(A\left(X_{s}(\omega), b\right)\right) \exp \left\{\left(\|\lambda\|^{2}-\|\rho\|^{2}\right)(t-s) / 2\right\} \quad P \text {-a.s. }
\end{aligned}
$$

From this one concludes as before that, for $x=\left(X_{0}, X_{t(1)}, X_{t(2)}, \ldots, X_{s}\right), \pi\left(x, d x_{n+1}\right)$ is $P$-a.s. the law of Brownian motion started from $X_{s}$ at time $t--s$. By induction one concludes that the joint distributions are those of Brownian motion.

REMARK 3.7. An alternate proof can be given without making any use of regular conditional probabilities or the key result of Proposition 3.4. It follows from Remark 3.5 that the "horocyclic" process $\left(H_{t}\right)_{t \geqslant 0}=\left(A\left(X_{t}, b\right)\right)_{t \geqslant 0}$ is Markov. Since conditional expectation commutes with the decomposition into plane waves (as 
$\left.E\left[\left|f_{b}\left(X_{t}\right)\right|\right] \leqslant C e^{-t\|\rho\|^{2} / 2}\right)$, one has that

$$
E\left[f\left(X_{t}\right) \mid \mathfrak{F}_{s}\right]=\int_{B} E\left[f_{b}\left(X_{t}\right) \mid \mathfrak{F}_{s}\right] d b=\int_{B} E\left[f_{b}\left(X_{t}\right) \mid X_{s}\right] d b=E\left[f\left(X_{t}\right) \mid X_{s}\right]
$$

This implies that $\left(X_{t}\right)_{t \geqslant 0}$ is Markov, cf. [1].

\section{BIBLIOGRAPHY}

1. R. Blumenthal and R. Getoor, Markov processes and potential theory, Academic Press, New York.

2. S. Helgason, Duality and Radon transform for symmetric spaces, Amer. J. Math. 85 (1963), 667-692.

3. Radon-Fourier transforms on symmetric spaces and related group representations, Bull. Amer.

Math. Soc. 71 (1966), 757-763.

4. __ Differential geometry, Lie groups, and symmetric spaces, Academic Press, New York, 1978.

5. __ Groups and geometric analysis, Academic Press, Orlando, 1984.

6. F. I. Karpelevic, The geometry of geodesics and the eigenfunctions of the Beltrami-Laplace operator on symmetric spaces, Trans. Moscow Math. Soc. 14 (1965), 51-199.

7. A. Koranyi and J. C. Taylor, Minimal solutions of the heat equation and uniqueness of the positive Cauchy problem on homogeneous spaces, Proc. Amer. Math. Soc. 94 (1985), 273-278.

Department of Mathematics, McGill University, Montreal, Québec, Canada H3A 2K6 